\title{
Kombinierte NDIR-PAS-Gasmessung mit großem Dynamikbereich und inhärenter Selbstüberwachung
}

\author{
Karsten Kühn, Eliseo Pignanelli und Andreas Schütze \\ Universität des Saarlandes, Lehrstuhl für Messtechnik (LMT), Saarbrücken, Campus A5.1
}

\section{Zusammenfassung}

Eine Kombination aus nicht-dispersiver Infrarot (NDIR) Transmissions- und photoakustischer (PA) Absorptionsmessung bietet neben den Prinzip bedingten Vorteilen eines physikalischen Messverfahrens (große Selektivität, hohe Genauigkeit und Stabilität) einen sehr großen Dynamikbereich und eine inhärente Selbstüberwachung durch die beiden komplementären Messverfahren. Basierend auf einem modular aufgebauten „low cost“-System können so z.B. $\mathrm{CO}_{2}$-Konzentrationen im Bereich von $50-20.000$ ppm bestimmt werden. Die erreichte Auflösung ist ausreichend, um z.B. eine robuste Überwachung der $\mathrm{CO}_{2^{-}}$ Konzentration in Innenräumen zu ermöglichen, als Basis für bedarfsgerechte Lüftungssteuerungen.

\section{Einleitung}

Die Gasmesstechnik wächst in ihrer Bedeutung als auch im Spektrum ihrer Anwendungen rasant. Ursachen hierfür sind unter anderem verschärfte Kriterien zum Umweltschutz oder auch neue Anwendungen wie z.B. die der Atemgasanalyse im Bereich der Medizin. Im Vordergrund des Nachweises einzelner Zielgase vor unerwünschten Hintergrundgasen steht dabei immer die Sensitivität, Selektivität als auch Stabilität des Messsystems. Die gewählte Kombination aus nicht-dispersiver Infrarotspektroskopie (NDIR) und photoakustischer Absorptionsspektroskopie (PAS) gewährt einen großen Dynamikbereich des Nachweises einzelner Zielgase bei gleichzeitiger Selbstüberwachung der Systemkomponenten.

\section{Experimentelles}

Das modular aufgebaute Gesamtsystem besteht aus einer adaptierbaren Messküvette, entsprechender Sensorik und einer dafür entwickelten Elektronik zum Auslesen der Messdaten als auch zur Konfiguration der ausgewählten Sensorik. Herzstück der Elektronik ist ein Mikrocontroller der Firma Atmel (AVR Atmega 2561), der die Konfiguration der Elektronik und die Datenakquise gewährleistet. Zur Bedienung des Gesamtsystems wurde eine graphische Benutzeroberfläche in LabVIEW programmiert, die gleichzeitig die Daten aufbereitet, um diese später mittels Matlab auswerten zu können.

\subsection{Hardware}

Als Strahlungsquelle wird ein moduliert betriebener, mikrostrukturierter Heizer (Intex, MIRL17-900) mit aufgesetztem Parabolspiegel zur Strahlbündelung und integriertem $\mathrm{CaF}_{2}$-Fenster genutzt. Zur $\mathrm{CO}_{2^{-}}$ Konzentrationsbestimmung ist ein gasspezifischer Filter mit einer zentralen Wellenlänge von 4,26 $\mu \mathrm{m}$ und einer Halbwertsbreite von 180 nm nachgeschaltet. Zur Messung der Transmission der zwischen 1 und $10 \mathrm{~Hz}$ einstellbaren, modulierten Strahlung durch die Gaskonzentration wird ein Thermopile-Detektor (Heimann Sensor, HIS) mit integriertem Verstärker genutzt. Die Absorption der elektromagnetischen Strahlung im Gas führt zu einem angeregten Zustand (erhöhte kinetische Energie der Moleküle) der Zielgaskomponenten und damit zu einer Temperaturerhöhung, die bei einem konstanten Volumen der Küvette eine Druckerhöhung zur Folge hat [1]. Durch die Modulation der IR-Strahlung resultieren in der Messküvette Druckschwankungen, die mit Hilfe von kapazitiven Mikrofonen (Knowles Acoustics, SPM0408HE5H) registriert und über eine 
Verstärkerschaltung inklusive Bandpass aufgezeichnet werden. Zur Überwachung der Umgebungsbedingungen sind in der Messküvette selbst noch ein Temperatur-/Feuchtesensor (Driesen + Kern, SHT75DK) sowie ein Drucksensor (AMSYS, ME707-002) zur Überwachung des statischen Drucks integriert. Bild 1 zeigt links schematisch das NDIR/PAS-Messsystem und rechts eine 3D-CAD Explosionszeichnung einer $9 \mathrm{~cm}$ langen Messküvette inklusive angeschlossener Sensorik. Die Messung erfolgt im „stopped flow“-Betrieb, d.h. die Küvette wird mittels zweier Ventile abgeschlossen, da sonst die Druckschwankungen durch Elastizitäten in den Zuleitungen deutlich reduziert und zusätzlich durch Strömungsrauschen überlagert würden.

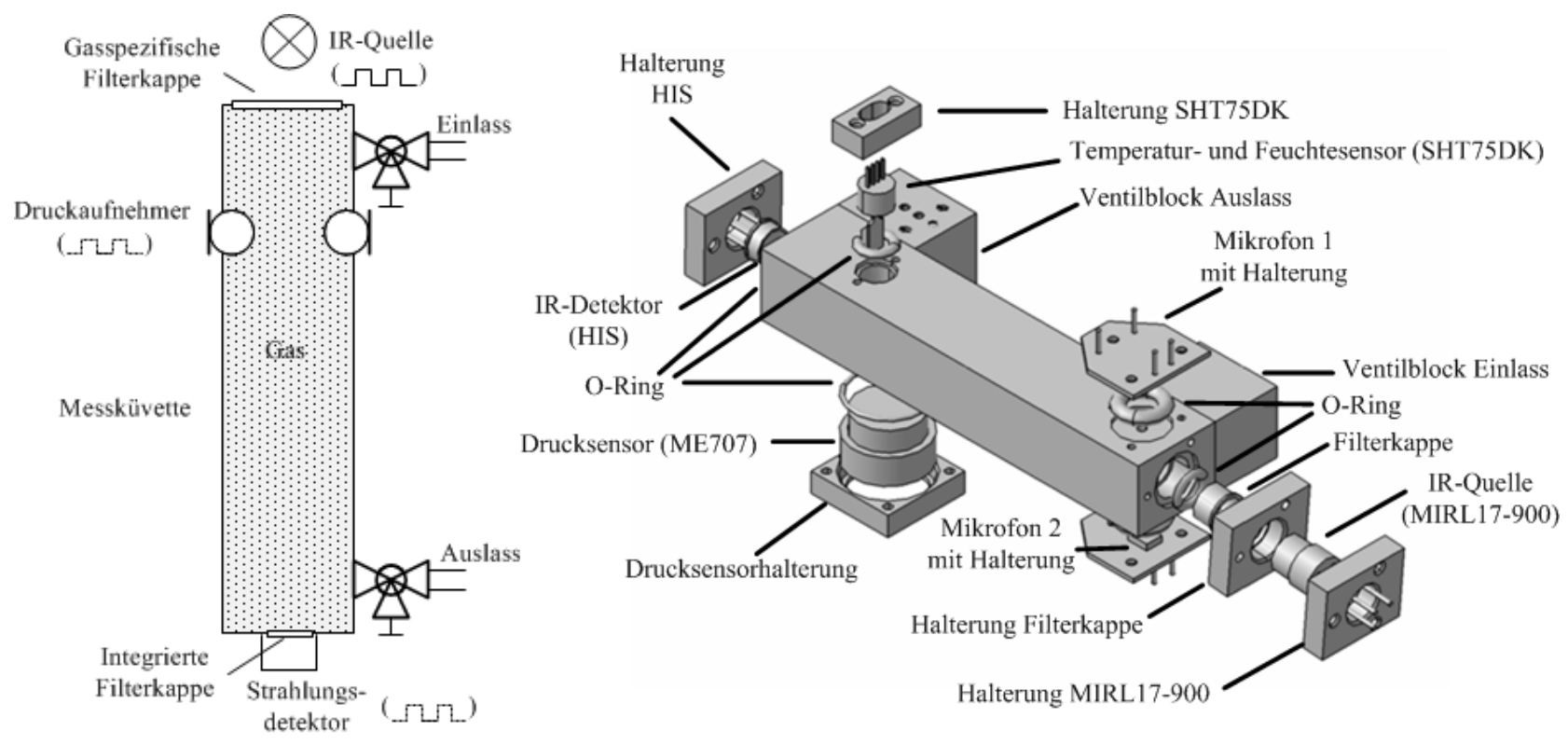

Bild 1 Kombiniertes NDIR/PAS-Messsystem: links Schema, rechts CAD-Modell der aus Edelstahl hergestellten Messküvette inklusive angeschlossener Sensorik [2].

\subsection{Signalverarbeitung}

Durch den Einsatz mikrostrukturierter IR-Quellen ohne einen zusätzlichen optischen Modulator (Chopper) ist eine Modulation zur Erzielung einer optimalen Modulationstiefe im unteren $\mathrm{Hz}$ Bereich möglich. Somit befindet sich die Messküvette in einem nicht-resonanten Modus, da die akustische Wellenlänge bei einer Frequenz zwischen 1 und $10 \mathrm{~Hz}$ viel größer als die optische Weglänge von $9 \mathrm{~cm}$ ist. Resonanzüberhöhungen durch entsprechend optimierte Messküvetten können also nicht genutzt werden, um ein besseres SignalRausch-Verhältnis zu erreichen.

Die Signalauswertung des NDIR/PAS-Messsystems basiert auf der Berechnung der diskreten Fouriertransformation (DFT) der aufgezeichneten Rohdaten von Thermopile bzw. Mikrofon. Der verwendete MatlabAlgorithmus zur Berechnung der einzelnen Fourierkomponenten entspricht dabei einer FFT (Fast Fourier Transformation). Zur weiteren Signalauswertung wird die Amplitude der Fourierkomponente der Anregungsfrequenz als Messwert zur Bestimmung der Zielgaskonzentration durch Aufnahme von Kalibrierkurven herangezogen. Ohne diesen Ansatz wäre eine Auswertung des PA-Signals für kleine Konzentrationen unmöglich, wie Bild 2 verdeutlicht. Während für die NDIR Rohdaten, dargestellt im linken Abschnitt für drei verschiedene $\mathrm{CO}_{2}$-Konzentrationen, z.B. eine Histogramm-Analyse der Amplitude des Signals realisierbar wäre, ist dies für die im rechten Abschnitt dargestellten Mikrofonsignale nicht möglich, da selbst bei einer Konzentration von 1.000 ppm das Signal kaum über dem Rauschen liegt. Die IR-Quelle wurde hierbei mit etwa $900 \mathrm{~mW}(7 \mathrm{~V}, 128 \mathrm{~mA})$ und $6 \mathrm{~Hz}$ rechteckförmig moduliert. Die Spiegelung des PAS Signals an dessen Mittelwert im Vergleich zum NDIR Signal ist bedingt durch die Elektronik des Messsystems. Ebenso ist der Mittelwert für beide Signale fest vorgegeben, um hinsichtlich des 10 bit ADC Modul des Mikrocontrollers eine optimale Auflösung zu gewährleisten. Basierend auf der Hauptkomponente der DFT (Amplitude der Anregungsfrequenz) wird die Gaskonzentration mit entsprechenden Kalibrierkurven sowohl für das NDIR- als auch PA-Signal bestimmt. Zusätzlich können beide Signale stets 
miteinander verglichen werden, um somit z.B. Veränderungen der Strahlungsintensität der benutzten IRQuelle oder eine Verschmutzung der Küvette bzw. der optischen Komponenten erkennen zu können, da beide Signale proportional zur IR-Intensität sind. Bei steigender Gaskonzentration sinkt das Transmissionssignal, während das PA-Signal steigt, so dass Konzentration und Intensität der Quelle prinzipiell unabhängig aus den beiden gemessenen Signalen bestimmt werden können.
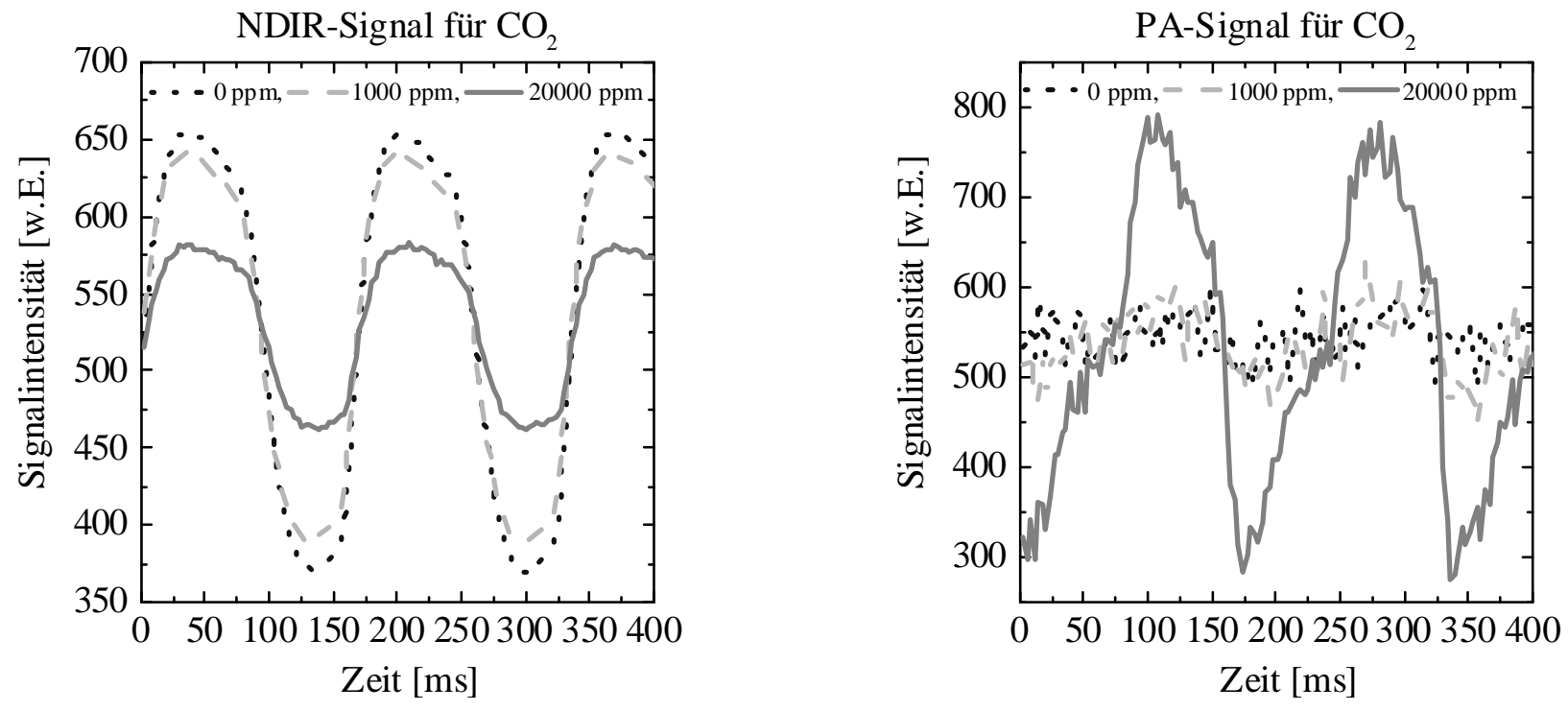

Bild 2 Gemessene Rohdaten für die IR-Transmission (links) bzw. das photoakustische Signal (rechts) für drei verschiedene $\mathrm{CO}_{2}$-Konzentrationen bei einer rechteckförmigen Modulation der IR-Quelle mit $6 \mathrm{~Hz}$.

\section{Ergebnisse}

Für die im Folgenden dargestellten Ergebnisse wurde die IR-Quelle jeweils rechteckförmig mit $6 \mathrm{~Hz}$ moduliert, um ein optimiertes Signal-Rausch-Verhältnis hinsichtlich der Verstärkerelektronik mit integriertem Bandpass zu erhalten. Bild 3 zeigt links die Amplitude der Fouriertransformierten des Thermopilesignals im Bereich der Anregungsfrequenz für unterschiedliche $\mathrm{CO}_{2}$-Konzentrationen und rechts die entsprechende Auswertung des Mikrofonsignals.
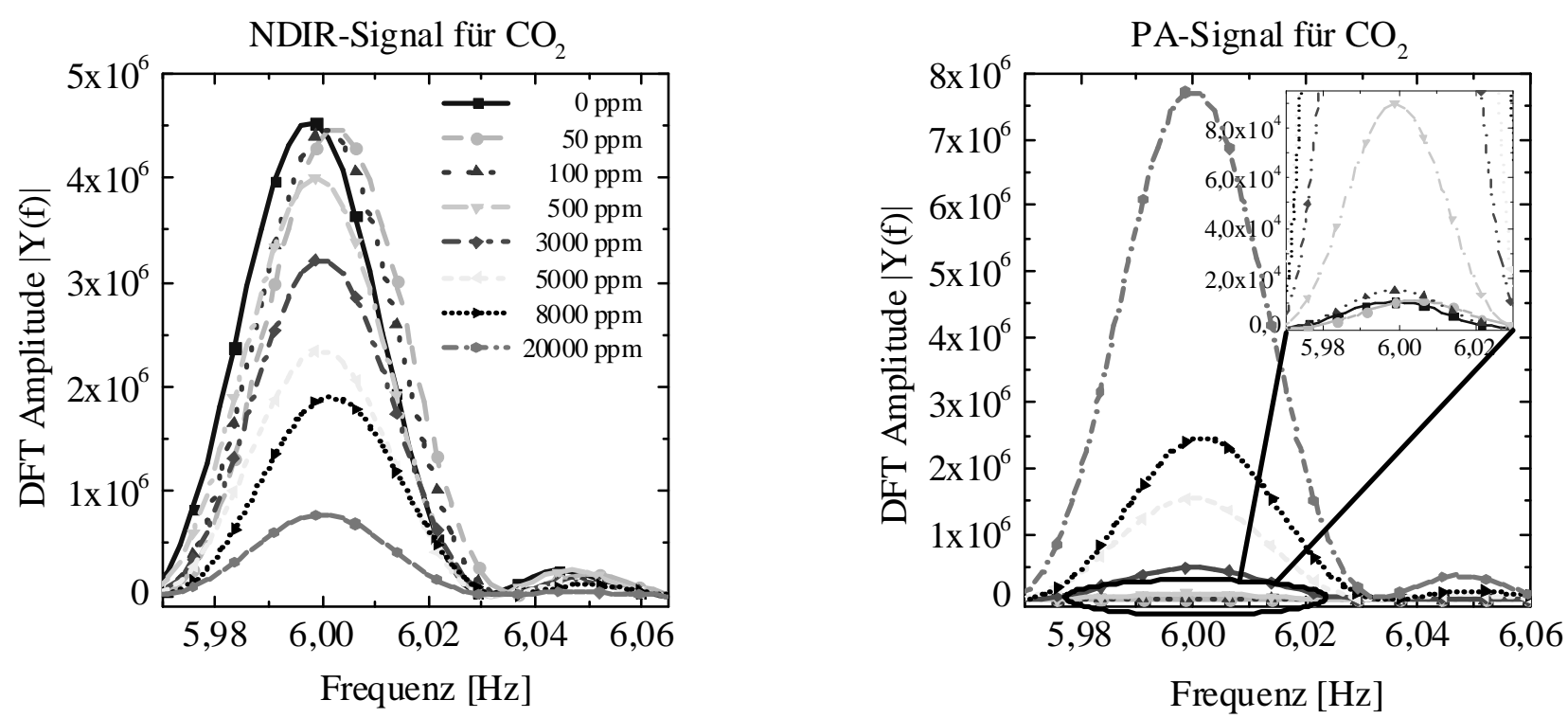

Bild 3 Ausschnitt aus dem DFT-Spektrum für Transmission (links) als auch PA-Signal (rechts) bei unterschiedlichen $\mathrm{CO}_{2}$-Konzentrationen.

In Bild 4 sind die Maximalwerte der DFT über der $\mathrm{CO}_{2}$-Konzentration sowohl für das NDIR- als auch das PA-Signal aufgetragen, links für kleinere Konzentrationen bei unterschiedlichen IR-Quellenspannungen. Als 
Fitfunktionen haben sich einfach exponentielle Funktionen (NDIR) bzw. Polynome zweiten Grades (PAS) bewährt. Zu erkennen sind die höhere Empfindlichkeit speziell für geringe Gaskonzentrationen als auch die größere Auflösung der PAS über den Konzentrationsbereich von 50 bis 20.000 ppm (entsprechend 2 \%). Im rechten Abschnitt von Bild 4 ist der Konzentrationsbereich von 0,2 bis $100 \% \mathrm{CO}_{2}$ (entsprechend 2.000 bis 1.000.000 ppm) abgedeckt. Hierbei fällt auf, dass ab Konzentrationen größer $5 \% \mathrm{CO}_{2}$ die Abhängigkeit des PA-Signals nicht weiter stetig ansteigt sondern abfällt. Hier könnte z.B. die Absorption binnen weniger mm nach Eintritt in die Messküvette abgeschlossen sein, so dass die lokalen Temperaturschwankungen sehr stark mit den Innenwänden der Messküvette wechselwirken und somit auch der Anteil der in Druckschwankungen resultierenden Energiebeiträge mit geringerer Absorptionstiefe abnehmen. Somit haben sich auch die üblichen Fitfunktionen leider nicht bewährt. Die Abweichungen der beiden in Bild 4 gezeigten Graphen in den überlappenden Konzentrationsbereichen bei 7 V Quellenspannung ist auf einen Umbau der Messküvette zurückzuführen.
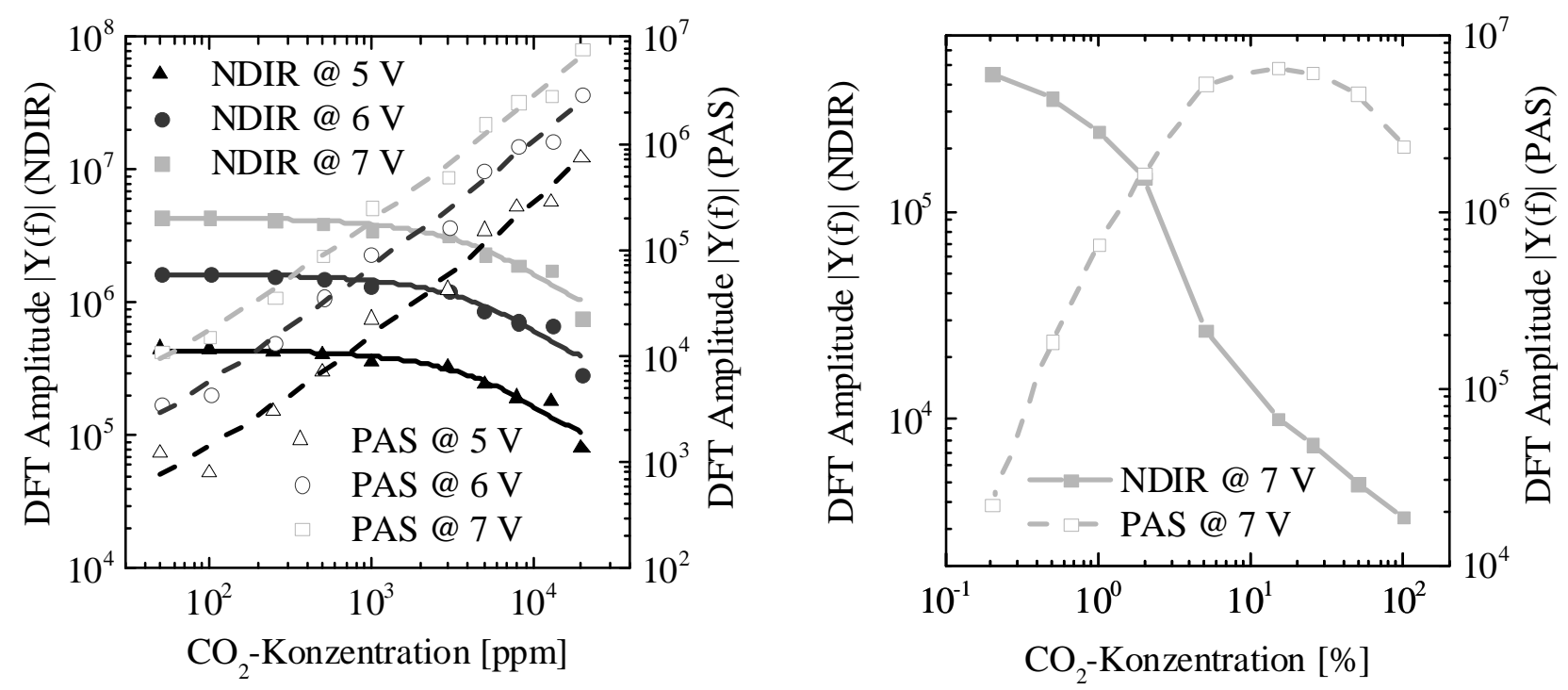

Bild 4 Kalibrierkurven (DFT Maximalwerte vs. Gaskonzentration) für unterschiedliche $\mathrm{CO}_{2}$-Konzentrationsbereiche. Links für drei unterschiedliche IR-Quellenspannungen für den Bereich von 50 bis $20.000 \mathrm{ppm}=2 \%$ (Messwerte und Fitfunktionen). Rechts bei 7 V Anregungsamplitude für den Konzentrationsbereich von 0,2 bis $100 \%$.

\section{Fazit und Ausblick}

Es wurde eine „low cost“ Kombination aus NDIR/PAS als Messsystem mit einem großen Dynamikbereich und inhärenter Selbstüberwachung des gesamten Systems vorgestellt. Hinsichtlich der vorgestellten Signalauswertung ist zu bemerken, dass diese individuell in Abhängigkeit des gewünschten Konzentrationsbereichs einer Applikation durch z.B. eine längere Absorptionsstrecke und/oder eine längere Messzeit für ein besseres Signal-Rausch-Verhältnis angepasst werden kann. Während sich PAS insbesondere für geringere Konzentrationen bewährt hat, eignet sich für höhere Konzentrationen das Transmissionssignal besser zur quantitativen Messung.

In Zukunft soll das Einsatzspektrum des Systems durch einen durchstimmbaren Fabry-Pérot Filter an Stelle der festen, durch einen Filter definierten Wellenlänge erweitert werden. Des Weiteren soll die IR-Quelle gegen einen temperaturgeregelten Halbleitergassensor mit passenden thermischen und optischen Eigenschaften getauscht werden, um die hohe Empfindlichkeit und das breite Ansprechspektrum eines Halbleitergassensors insbesondere im temperaturzyklischen Betrieb mit der Präzision der IR-Messtechnik kombinieren zu können.

\section{Literatur}

[1] J. Staab: Industrielle Gasanalyse. München: Oldenburg Verlag, 1994.

[2] E. Pignanelli: Gas-Konzentrationsbestimmung mittels kombinierter transmissions- und absorptionsspektroskopischer IR-Analyse. Saarbrücken: Diplomarbeit, LMT (Universität des Saarlandes), 2011. 